\title{
A chip embedding solution based on low-cost plastic materials as enabling technology for smart labels
}

\author{
Maarten Cauwe ${ }^{1}$, Bjorn Vandecasteele ${ }^{1}$, Johan De Baets ${ }^{1}$, Jeroen van den Brand ${ }^{2}$, \\ Roel Kusters ${ }^{2}$, Ashok Sridhar ${ }^{2}$ \\ ${ }^{1}$ imec-Cmst, Technologiepark 914a, B-9052 Zwijnaarde, Belgium \\ ${ }^{2}$ Holst Centre/TNO, HTC31, Postbus 8550, 5605 KN Eindhoven, the Netherlands. \\ Tel.: +32-9-2645353, Fax: +32-9-29645374, Maarten.Cauwe@imec.be
}

\begin{abstract}
Expanding the current smart packaging solutions to individual products requires improvement for several of the following properties: cost, thickness, weight, flexibility, conformability, transparency, and even stretchability. This paper focusses on an embedding technology that targets the first four properties, with an emphasis on cost reduction. The progress on the development work for this foil-based embedding is reported, along with a detailed failure analysis of the process flow. A functional demonstrator is realized in the form of a smart sensing label, including an embedded micro controller. Practical and technological shortcomings of the current technology are used as a starting point for proposing an improved embedding technology based on lowcost plastic materials. The first developments for lamination and via interconnection are described.
\end{abstract}

\section{Introduction}

Smart packaging aims at the integration of intelligence into the packaging of everyday products. For example, smart sensor foils integrated into food packages could benefit consumers by ensuring freshness and quality, while allowing the retail industry to manage food stocks and product authenticity more efficiently. These smart systems need to be ultra-small, cheap, intelligent, and low-power and include sensors, actuators, and processing and communication abilities, often in a single-package wireless solution. Commercially available solutions for smart packaging applications focus on temperature or humidity sensing and shock detection. The products are quite bulky and relatively costly (between $€ 10$ and $€ 100$ ), and as such are used to track complete pallets rather than individual packages or products. Reducing the cost and form factor of these smart labels is the prime requirement for introducing this concept at the product level [1].

Recently, a foil-based chip embedding technology was jointly developed by Holst Centre and imec-Cmst [2]. In contrast to fan-out WLP [3] and chip embedding in rigid or flexible printed circuit boards [4, 5], where cost reduction is achieved by scaling to larger panel sizes, low-cost was a main development goal of this chip embedding technology. The roll-to-roll compatible process flow starts by placing a chip on a bare copper foil using anisotropic conductive adhesive. The actual embedding is performed by laminating a PET film onto the copper foil with the chips, using a suitable adhesive. In the final step, the copper is structured, resulting in a thin $\mathrm{Cu}$ based circuitry foil with embedded components. The advantages of this approach in comparison to other hybrid integration methods based on low-cost materials are the removal of temperature limitations for die bonding, mechanical and physical protection of the chip and the realization of a flat surface which allows for direct access to the contacts of the chip. Using this foil-based embedding technology to realize smart sensing labels offers a clear cost reduction in combination with a smaller form factor and increased flexibility.

This paper starts with an overview of the single-layer embedding technology, focussing on the progress that is made since the previous publication [2]. To demonstrate the capabilities of the foil-based embedding, a smart label demonstrator with an embedded micro controller is realized. A failure analysis is performed on the test vehicle and demonstrator manufacturing process, revealing some remaining challenges. Based on the experience from the single-layer embedding, an improved embedding technology is proposed offering double-layer routing for more complex circuitry and passive component embedding for realizing a completely flat system-in-foil.

\section{Single-layer embedding technology}

The process flow starts with a single copper foil, including laser-drilled markings for the alignment of the following process steps. The first step of this embedding technology is the application of the die bonding adhesive. To be compatible with ultrafine-pitch applications, an anisotropic conductive adhesive is used in combination with $\mathrm{Ni} / \mathrm{Au}$ bumps on the chips. The die bonding adhesive is applied by dispensing to ensure a complete filling of the die bond adhesive underneath the chip with a minimum fillet at the edge and a uniform thickness after placement. During placement of the thinned chips, the bumps on the chip are aligned to markers on the foil.

The actual embedding of the chips is performed in a lamination step using PET film and a suitable adhesive ${ }^{1}$. Void-free lamination, ensuring a good encapsulation of the chips, is a crucial step in the chip embedding process. Due to the combination with PET film, the lamination temperature is limited to a maximum of $120{ }^{\circ} \mathrm{C}$ for a few minutes. The adhesive needs to combine good adhesion to copper and PET with a good flow behavior to successfully enclose the chips. In a final step, the copper is structured using conventional $\mathrm{PCB}$ processes or alternative patterning technologies to define the circuitry. The end result is a thin foil with embedded

1 Unless explicitly mentioned otherwise, the word "adhesive" in the following always refers to the adhesive used to encapsulate the chips. 
components and copper interconnects. Figure 1 shows a schematic overview of the process flow. Typical dimensions are a chip thickness of $30 \mu \mathrm{m}$, a $20 \mu \mathrm{m}$ die bond adhesive layer, and a $50 \mu \mathrm{m}$ thick PET film. The adhesive surrounding the chips will be about $70 \mu \mathrm{m}$ to $100 \mu \mathrm{m}$ in total, resulting in an overall thickness of less than $200 \mu \mathrm{m}$.

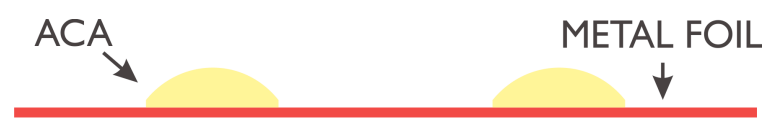

1. ADHESIVE DISPENSING

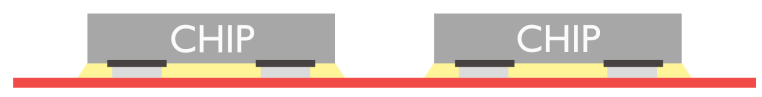

2. CHIP PLACEMENT

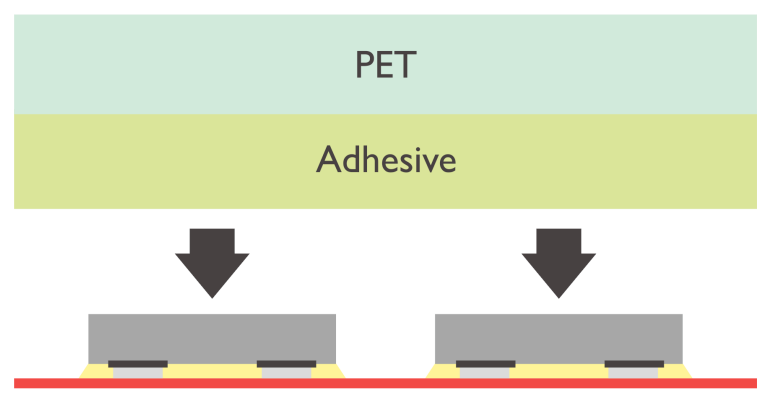

3. LAMINATION

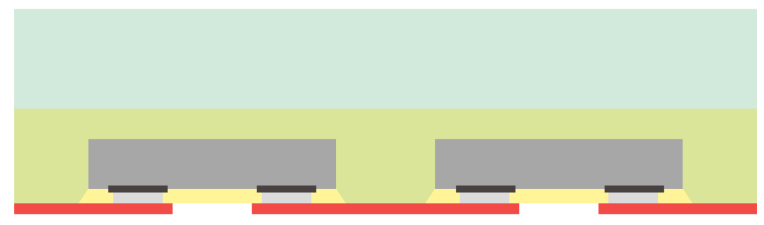

\section{STRUCTURING}

Figure 1 Process flow for foil-based chip embedding

The ideal adhesive for this technology would be a film adhesive which becomes liquid at a temperature below $80{ }^{\circ} \mathrm{C}$, flows around the chips and subsequently solidifies. This could be either a thermoplastic or thermosetting polymer, although the latter would accommodate a low processing temperature without restricting the operating temperature of the circuitry.

A significant number of different general-purpose industrial adhesive types are evaluated, ranging from pressure sensitive tapes, over thermoplastic or thermosetting film adhesives, to UV curing liquid adhesives. Table 1 gives an overview of the different types of adhesive on processability, cost, roll-to-roll compatibility of the process flow and embedding performance. Overall, the pressure-sensitive tapes and the heat-activated films rank the best. The embedding performance of the thermoplastic adhesives is very good, but to achieve a good flow around the chip in an acceptable time, temperatures above the limit for PET are required. Both the pressure-sensitive and the heat-activated adhesive need a higher thickness than originally anticipated. For PSAs a thickness of twice the height of the chip and the die attach adhesive is recommended, while for the heat-activated material, a margin of $30 \mu \mathrm{m}$ above the chip is sufficient. For the specified thickness of the chips and the die attach adhesive, an adhesive thickness of $100 \mu \mathrm{m}$ is optimal.

Table 1 Comparison of the different types of adhesive on processability, cost, roll-to-roll compatibility of the process flow and embedding performance

\begin{tabular}{|l|c|c|c|c|}
\hline Material & Proc. & Cost & R2R & Perf. \\
\hline PSA & + & ++ & ++ & + \\
\hline HAA & ++ & + & ++ & ++ \\
\hline TP & -- & + & - & ++ \\
\hline Liquid & - & - & + & + \\
\hline
\end{tabular}

To evaluate the embedding process and to verify the reliability of the interconnections, a technology demonstrator is designed, consisting of nine embedded daisy chain test chips that are interconnected to create a continuous daisy chain. Two four-point measurement test structures are foreseen per chip, making it possible to accurately measure the contact resistance between the bumps on the chip and the copper pads.

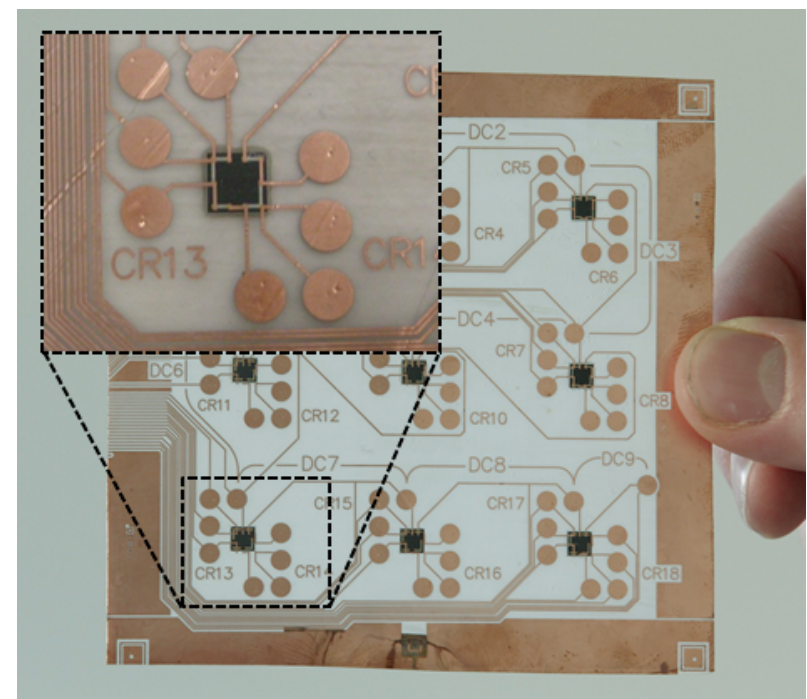

shows a top view of one of the realized technology demonstrators and Figure 2 a cross section of one of the embedded chips. A total of eight samples are manufactured to determine the critical steps in the process flow and evaluate the failure mechanism during stress testing. Half of the samples are laminated using the selected heat-activated adhesive and a pressure-sensitive tape adhesive is applied to the other half. Due to the limited amount of available chips, not all samples were fully populated, resulting in 28 chips or 280 contacts per material. After each process step the integrity of the samples is verified by optical inspection. The contact resistance to the chip is measured on the final samples using a four-point measurement technique and these measurements are repeated after stress testing of the interconnects (thermal cycling from $-40{ }^{\circ} \mathrm{C}$ to $85{ }^{\circ} \mathrm{C}$ and temperature-humidity storage at $\left.85{ }^{\circ} \mathrm{C} / 85 \% \mathrm{RH}\right)$. 


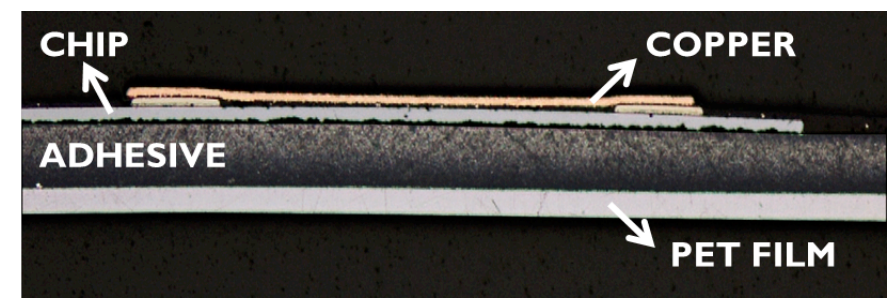

Figure 2 Cross section of an embedded test chip

A failure analysis of the single-layer embedding process revealed a number of different failure mechanisms with varying impact. A minor issue, for which no direct impact on the performance could be determined, was the entrapment of air after lamination. At the edge between the die attach adhesive and the copper foil, tiny bubbles appeared after lamination, which are most likely caused by entrapped air at the irregular edge of the die attach adhesive. The two most important failure mechanisms while processing are chip cracking during assembly of the ultra-thin chips and misalignment after copper structuring. Optimization of the pressure during die bonding can significantly reduce the amount of cracked chips, but slightly increasing the chip thickness to $50 \mu \mathrm{m}$ offers a good compromise between processability and overall thickness. Alignment issues are overcome by optimizing the design and location of the alignment markers.

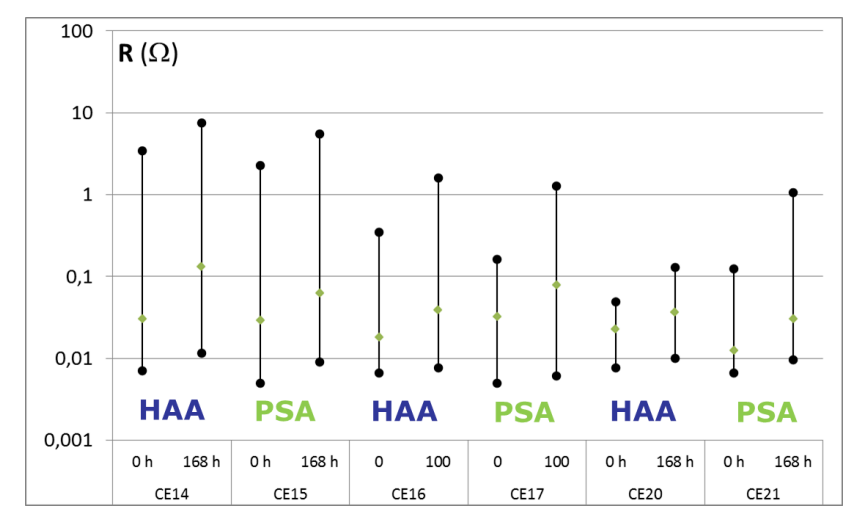

Figure 3 Contact resistance measurements before and after stress testing (CE14, 15, 20, 21: 168 hours at $85^{\circ} \mathrm{C} / 85$ $\%$ RH; CE16, 17: 100 cycles of $-40^{\circ} \mathrm{C}$ to $85^{\circ} \mathrm{C}$ )

Comparing the contact resistance measurements before and after stress testing (Figure 3), shows an overall increase in contact resistance for all chips on all samples (CE14, 15, 20, 21: 168 hours at $85{ }^{\circ} \mathrm{C} / 85 \% \mathrm{RH}$; CE16, 17: 100 cycles of $40{ }^{\circ} \mathrm{C}$ to $85{ }^{\circ} \mathrm{C}$ ). The increase in resistance is related to the presence of copper oxide at the interface between the ACA and the copper foil, which grows during stress testing. The copper oxide is a natural result of the surface treatment of the copper foil to obtain a higher adhesion to the embedding adhesive and thus a compromise between adhesion and contact corrosion will have to be made.

To resolve this weak point observed during stress testing, the impact of the copper foil treatment on the adhesion is investigated. A comparison of the peel strength between the copper and the adhesive is made using copper foils with different surface treatments. Regardless of the treatment, all peel strengths are in the range of $1.0-1.5 \mathrm{~N} / \mathrm{mm}$, so an untreated, electrodeposited copper foil (Circuit Foil $18 \mu \mathrm{m} \mathrm{BF}$ $P L S T P)$ is chosen for further processing. The effect on the contact reliability is currently under investigation.

\section{Smart label demonstrator}

As mentioned in the introduction, cost-effective sensor patches for use in a variety of smart packaging applications are a natural target for the foil-based embedding technology. The demonstrator of choice is a versatile smart sensing label that can be combined with a humidity, amine or ethylene sensor. The system design is the result of collaboration between the $\mathrm{SiF}$ and the WATS sensor program at the Holst Centre in Eindhoven [6]. The smart label consists of a microcontroller to perform the measurements, translate the electrical signals into data and store the data with a time stamp in a memory; together with a NFC radio chip that can transmit the data to most recent smart phones or to a dedicated reader. Next to the chips, a number of passive components, an integrated antenna, a flexible battery and the interface to the sensing foil are also part of the smart label.

To demonstrate the capabilities of the foil-based embedding technology, the micro controller (a Texas Instruments MSP430F1611) is chosen for embedding. The die size is $4.6 \mathrm{~mm}$ by $4.4 \mathrm{~mm}$ and the minimum pad pitch is 80 $\mu \mathrm{m}$. The remaining components, including the NFC radio chip in a QFN package, are surface mounted using isotropic conductive adhesive. During handling of the technology demonstrator, it became clear that the stickiness and softness of the pressure-sensitive adhesive could lead to difficulties with assembly of surface-mount components. In view of this, the smart label demonstrator is only realized using the heatactivated adhesive.

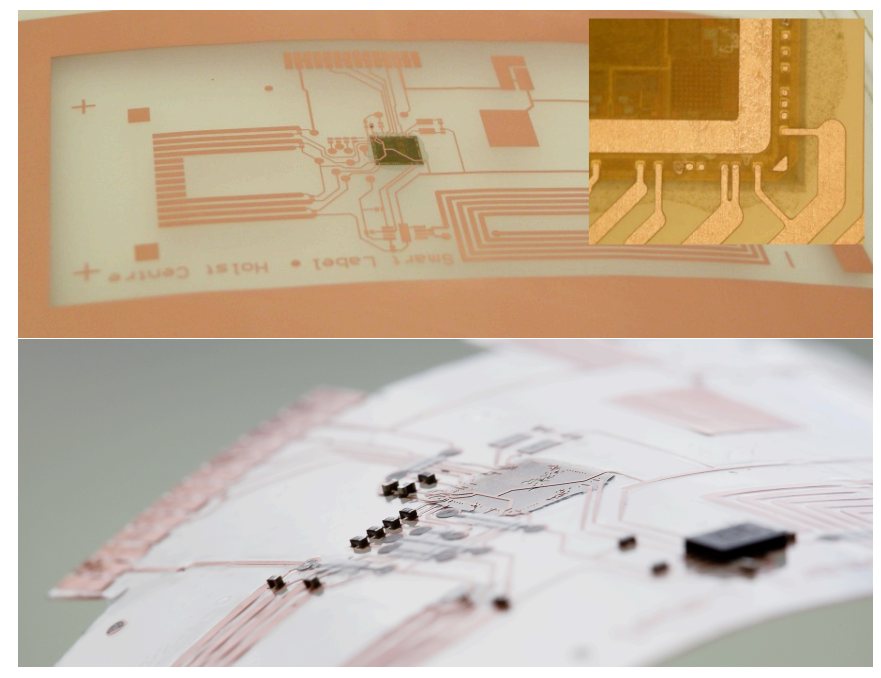

Figure 4 Overview of the smart label demonstrator

Figure 4 shows an overview of the functional smart label demonstrator. The embedded micro controller is clearly visible in the centre of the top picture, with the interface to the sensing foil on the left and the antenna in the bottom right corner (partially hidden by the inset). The inset shows a closeup of the interconnections to the chip, illustrating the low pad pitch on the micro controller die. The lower picture highlights the flat surface above the embedded chip, emphasized by the 
protruding passive components and the QFN package of the NFC chip on the right.

\section{Double-layer embedding technology}

The foil-based embedding technology that was discussed up till now, which will be further referred to as "single-layer embedding technology", made it possible to embed thinned chips in a foil-based system of less than $200 \mu \mathrm{m}$ thick. Due to the limitation in adhesive thickness, it is not possible to embed thicker components, such as passives. This is a major restriction on the applicability of this technology, as the advantage of a flat and accessible surface are nullified by mounting the necessary passive components. Complex systems-in-foil may also require more than one signal layer, another limitation of the current technology.

Apart from these practical reasons, there are also some technological limitations. The heat-activated adhesive used for embedding is quite soft and remains somewhat sticky, even after full curing. This causes the copper traces, which are held together mechanically by the adhesive, to be quite prone to movement during further processing. Mounting components using thermocompression, for examples, is not compatible with the current approach.

In view of these limitations, a new approach to chip embedding based on low-cost plastics is presented. The double-sided circuitry embedding technology is an extension of the single-sided, foil-based chip embedding, where the PET film is replaced by a second metal foil. To obtain sufficient mechanical strength and to further reduce cost, the adhesive film is replaced by a substrate material which is compatible with the chip embedding concept. The process flow for double-sided embedding, shown in Figure 5, is very similar to the single-sided technology. In the first step the components are mounted on a metal foil using isotropic or anisotropic conductive adhesive. Next to naked dies, also thin passive components are embedded so the outer surface is completely flat. Instead of laminating a PET film with a suitable adhesive, the embedding material now acts as a substrate layer, with a metal circuit layer on both sides. The material used for embedding the chips can be any kind of polymer that is cast, coated or laminated onto the metal foil, depending on the requirements of the final application. Interconnections between the two metal foils are realized by laser drilling and metallization of the vias. The preferred option for the latter is filling the vias with conductive paste, a technology that was developed at the Holst Centre in Eindhoven [7]. Alternatives are plating or some form of crimping.

Next to a good flow for embedding and sufficient adhesion to the metal foil, the selection criteria for substrate materials also include the necessary properties for serving as a substrate for flexible electronic circuitry. Depending on the application, dimensional stability, thermal resistance, tear resistance and flexibility can be specified. All these parameters, along with the material cost, should be taken into account during the material selection. The method for applying the material to the metal foil with the chips depends on the nature of the polymer material. Thermoplastic materials can be cast in liquid form, but this requires drawing of the substrate material in two directions to obtain sufficient dimensional stability. Coating of thermosetting materials is also a possibility, although this is more suited for applying very thin layers. Laminating film materials (thermoplastic or thermosetting) remains the most straightforward option.

\section{Component placement on copper foil}

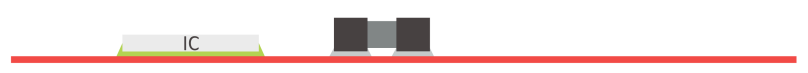

\section{Embedding}

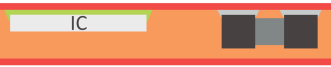

\section{Structuring}

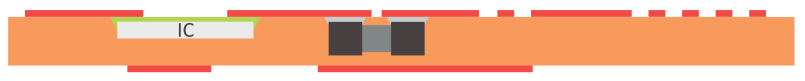

\section{Via drilling}

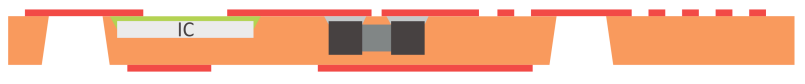

5. Via filling

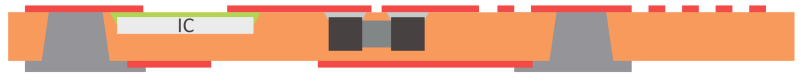

Figure 5 Process flow for double-sided chip embedding

During the development of the single-layer embedding technology, a large number of different material types were evaluated to determine the most suitable adhesive. For the double-layer embedding, the requirements for the substrate material are strongly linked to the application. For the smart label, the goal is to develop a flat system-in-foil, which can be laminated onto a flexible battery. The mechanical stiffness of the substrate is thus less important. As such, the first experiments are started using available film materials and applying these materials by lamination. From the Cmst research on stretchable electronics [8], a number of thermoplastic polyurethane (TPU) films are available in varying thickness. A selection of these films, namely Epurex Platilon U2102, U4201 and U073, are subjected to the IPCTM-650-2.3.17.1b flow test (Resin Flow of Adhesive Coated Films and Unsupported Adhesive Films). The results of the first trials showed excessive squeeze-out of the material, probably caused by too high lamination temperature and pressure during the first trials. Further experiments revealed that the Platilon U2102 material seemed the most promising for the embedding technology.

The lamination parameters for the selected Platilon U2102 material are optimized in two steps. First, the IPC-TM-6502.3.17.1b flow test is repeated with varying lamination time and pressure at a fixed temperature of $160^{\circ} \mathrm{C}$, which is at the upper end of the softening range of the TPU material. The results show that the lamination time has more influence on the flow than the pressure, but a higher pressure can give more flow for short lamination times. The second part of the lamination parameter optimization is performed using dummy silicon chips of $50 \mu \mathrm{m}$ thickness mounted on a $20 \mu \mathrm{m}$ die attach tape. A $100 \mu \mathrm{m}$ Platilon U2102 AK TPU film is placed in between the copper foil with the chips and another copper 
foil and laminated for $5 \mathrm{~min}$ at $160{ }^{\circ} \mathrm{C}$ and a pressure of $5 \mathrm{bar}$ or for $2 \mathrm{~min}$ at $170{ }^{\circ} \mathrm{C}$ and a pressure of 7 bar. Both parameter sets result in a good flow around the chips without voids (Figure 6), although some entrapped air bubbles are detected at the edge of the chip. Laminating at a lower temperature (easier handling) and pressure (less risk of chip cracking) is preferred at the expense of a longer lamination time. The same lamination parameters are used to embed $150 \mu \mathrm{m}$-thick passive components inside a $250 \mu \mathrm{m}$ Platilon U2102 AK TPU film (Figure 7).

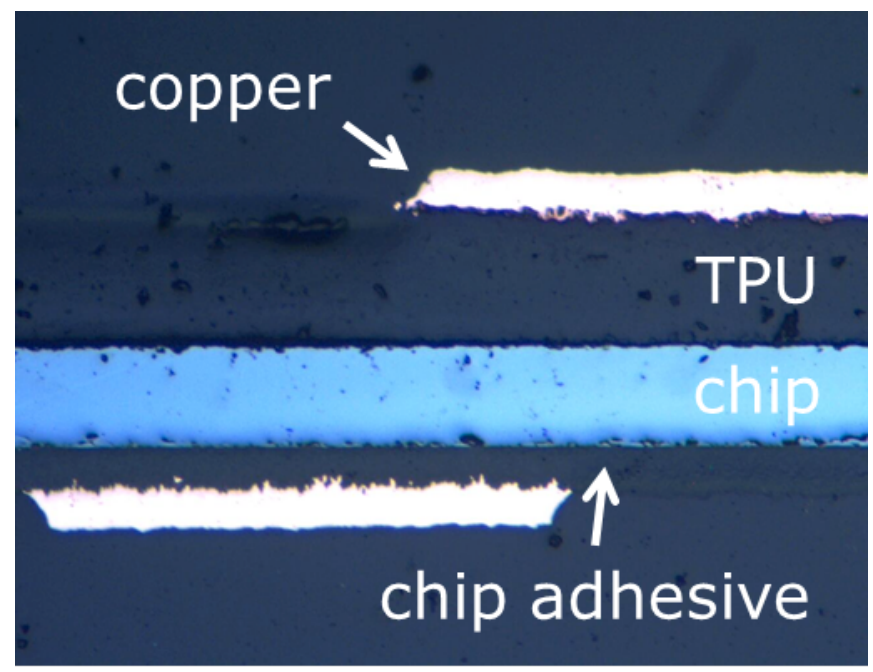

Figure 6 Cross section of a dummy silicon chip (50 $\mu \mathrm{m}$ thickness) embedded in a thermoplastic polyurethane substrate between two copper layers

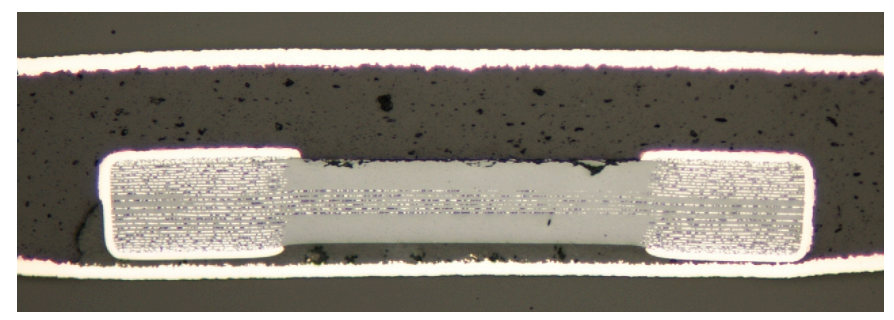

Figure 7 Cross section of a $150 \mu$ m-thick passive component embedded in a thermoplastic polyurethane substrate between two copper layers

Interconnections between the two copper layers are realized by drilling via holes through the TPU substrate and subsequently filling these vias with a conductive paste. The via drilling is performed using a $\mathrm{CO}_{2}$ laser, possibly followed by a cleaning step using a $\mathrm{KrF}$ excimer laser. A parameter investigation is performed to determine the optimal fluence and number of pulses to drill the vias. For the selected TPU film, an ablation rate of $20 \mu \mathrm{m}$ per pulse at a fluence of 12 $\mathrm{J} / \mathrm{cm}^{2}$ is achieved. The subsequent cleaning with the excimer laser requires about 10 pulses but at a much lower fluence of $200 \mathrm{~mJ} / \mathrm{cm}^{2}$.

The process for via filling that was developed at the Holst Centre has already proven its functionality and is thus the method of choice for realizing the via interconnections [6]. The conductive paste needs to combine a low viscosity for via filling with ensuring a reliable, low-resistance contact to both copper layers. An extensive design of experiment for determining the optimal parameters is still ongoing.

The double-layer embedding technology is very versatile, as it is compatible with a broad range of polymer materials, for which the specifications can be tuned to the final application. By completely embedding all of the components into the substrate, mounting this system-in-foil to other subsystems becomes easier. The direct access to the contacts of the chip also helps to reduce the complexity of interconnecting multiple subsystems, such as power generating foils, sensor nodes or flexible displays.

\section{Conclusions}

The progress on the development work for a foil-based embedding technology is described in this paper. A failure analysis of the single-layer embedding process revealed that the chip placement and the alignment of the copper pattern are the most critical steps. The reliability of the interconnection to the chip is hindered by the copper oxide present as a result of the surface treatment of the copper foil. A solution of using an untreated foil is proposed, but remains untested.

A functional demonstrator is realized in the form of a smart label, capable of wireless monitoring of temperature and humidity. The micro controller chip, with a minimum pad pitch of $80 \mu \mathrm{m}$, is successfully embedded.

Realizing the technology and functional demonstrators using the single-layer embedding technology revealed a number of shortcomings of the current approach, such as the restriction on component thickness or the mechanical properties of the adhesive. To overcome these issues, a new embedding approach is proposed, including passive component embedding and double-layer routing. The feasibility of this approach is demonstrated by a number of short trials. This new approach will make it possible to realize a complete flat, $250 \mu \mathrm{m}$ thick, flexible label, which can be easily laminated onto a flexible battery.

\section{Acknowledgments}

The authors would like to thank Marc Koetse and Jeroen Schram for their aid in realizing the smart label demonstrator and Bart van Remoortere for the via filling experiments.

\section{References}

1. Briand, D, Molina-Lopez, F, Vásquez Quintero, A, Ataman, C, Courbat, J, and de Rooij, N, "Why Going Towards Plastic and Flexible Sensors?", Procedia Engineering, Vol. 25 (2011), pp. 8-15.

2. Cauwe, M, Vandecasteele, B, Gielen, A, De Baets, J, van den Brand, J, and Kusters, K, "Technology development for a low-cost, roll-to-roll chip embedding solution based on PET foils," Proc $18^{\text {th }}$ European Microelectronics and Packaging Conf, Brighton,UK, Sep. 2011, pp. 559-564.

3. Brunnbauer, M, Furgut, E, Beer, G, and Meyer, T, "Embedded wafer level ball grid array (eWLB)," Proc $8^{\text {th }}$ Electronics Packaging Technology Conf, Singapore, Dec. 2006, pp. 1-5.

4. Ostmann, A, Manessis, D, Stahr, J, Beesley, M, Cauwe, M, and De Baets, J, "Industrial and Technical Aspects of Chip Embedding Technology," Proc $2^{\text {nd }}$ Electronics System-Integration Technology Conf, Greenwich, UK, Sep. 2008, pp. 315-320. 
5. Christiaens, W, Loeher, T, Pahl, B, Feil, M, Vandevelde, $\mathrm{B}$, and Vanfleteren, J, "Embedding and assembly of ultrathin chips in multilayer flex boards," Circuit World, Vol. 34 (2008), pp. 3-8.

6. Koetse, M, "Smart Systems, integration of classic and plastic electronics on a system level", Proc $5^{\text {th }}$ Large-area, organic and printed electronics conv, Munich, Jun. 2012.

7. van den Brand, J, Saalmink, M, Barink, M, Dietzel, A, "Novel lamination and interconnection technologies demonstrated in a flexible modular optical sensor array for wound monitoring," Microelectronic Engineering, Vol. 87 (2010), pp. 769-772.

8. Vanfleteren, J, Gonzalez, M, Bossuyt, F, Hsu, YY, Vervust, T, De Wolf, I, Jablonski, M, "Printed circuit board technology inspired stretchable circuits," $M R S$ BULLETIN, Vol. 37 (2012), pp. 254-260. 